\title{
SURGERY FOR ACQUIRED HEART DISEASE
}

\section{GUIDELINES FOR REPORTING MORBIDITY AND MORTALITY AFTER CARDIAC VALVULAR OPERATIONS}

L. Henry Edmunds, Jr., MD, Richard E. Clark, MD, Lawrence H. Cohn, MD, Gary L. Grunkemeier, PhD,

D. Craig Miller, MD, and Richard D. Weisel, MD*

A the request of the Councils of The Society of Thoracic Surgeons (STS) and The American Association for Thoracic Surgery (AATS), the Ad Hoc Liaison Committee for Standardizing Definitions of Prosthetic Heart Valve Morbidity "revisited" the Guidelines published in September 1988..$^{1-3}$ The purpose of the review was to update and clarify definitions within the guidelines and to consider recommendations made by others. ${ }^{4,5}$ The variety of cardiac valvular procedures has expanded since 1988; therefore, in this document the term operated valve indicates prosthetic and bioprosthetic heart valves of all types, operated or repaired native valves, and allograft and autograft valves. The term operated valve includes any cardiac valve altered by a surgeon during an operation.

Much morbidity and mortality is a direct consequence of the interaction between the patient and operated valve(s), although patient variables (e.g., age, degree of coronary arterial disease, follow-up care) may be more responsible for outcomes than an operated valve. However, no set of guidelines can identify all possible patient factors that may affect morbidity and mortality. General agreement regarding the following definitions of terms and suggestions for reporting data does not preclude more detailed analyses or constructive recommendations, and investigators are encouraged to identify relevant patient factors in addition to factors related to operated valves.

This article is being published concurrently in The Journal of Thoracic and Cardiovascular Surgery, The Annals of Thoracic Surgery, and the European Joumal of Cardio-Thoracic Surgery.

*Members, Ad Hoc Liaison Committee for Standardizing Definitions of Prosthetic Heart Valve Morbidity of The American Association for Thoracic Surgery and The Society of Thoracic Surgeons.

J Thorac Cardiovase Surg 1996;112:708-11

Copyright (C) 1996 by Mosby-Year Book, Inc.

$0022-5223 / 96 \$ 5.00+0 \quad \mathbf{1 2 / 1 / 7 4 9 0 8}$

\section{Purpose}

The purpose of these guidelines is to facilitate the analysis and reporting of results of operations on diseased cardiac valves. The definitions and recommendations that follow are guidelines, not standards, and are designed to facilitate comparisons between the experiences of different surgeons who treat different cohorts of patients at different times with different techniques and materials.

\section{Mortality}

Thirty-day mortality (sometimes termed operative mortality) is death within 30 days of operation regardless of the patient's geographic location. Follow-up for 30-day mortality must be complete. Hospital mortality is death within any time interval after operation if the patient is not discharged from the hospital. Hospital to hospital transfer is not considered discharge; transfer to a nursing home or rehabilitation unit is considered hospital discharge unless the patient subsequently dies of complications of the operation.

\section{Definitions of morbidity}

Structural valvular deterioration (SVD). Any change in function (a decrease of one New York Heart Association functional class or more) of an operated valve resulting from an intrinsic abnormality of the valve that causes stenosis or regurgitation.

Structural valvular deterioration includes operated valve dysfunction or deterioration exclusive of infection or thrombosis as determined by reoperation, autopsy, or clinical investigation. The term structural deterioration refers to changes intrinsic to the valve, such as wear, fracture, poppet escape, calcification, leaffet tear, stent creep, and suture line disruption of components (e.g., leaflets, chordae) of an operated valve.

Nonstructural dysfunction. Any abnormality resulting in stenosis or regurgitation at the operated valve that is not intrinsic to the valve itself. 
Nonstructural dysfunction refers to nonstructural problems that result in dysfunction of an operated valve exclusive of thrombosis and infection diagnosed by reoperation, autopsy, or clinical investigation. Examples of nonstructural dysfunction include entrapment by pannus, tissue, or suture, paravalvular leak, inappropriate sizing or positioning, residual leak or obstruction from valve implantation or repair, and clinically important hemolytic anemia.

Sudden or progressive dysfunction or deterioration of the operated valve may be structural, nonstructural, or both as determined by reoperation, autopsy, or clinical investigation.

Valve thrombosis. Any thrombus, in the absence of infection, attached to or near an operated valve that occludes part of the blood flow path or that interferes with function of the valve.

Valve thrombosis may be documented by operation, autopsy, or clinical investigation.

Embolism. Any embolic event that occurs in the absence of infection after the immediate perioperative period (when anesthesia-induced unconsciousness is completely reversed).

A neurologic event includes any new, temporary or permanent, focal or global neurologic deficit. A transient ischemic attack (TLA) is a fully reversible neurologic event that lasts less than 24 hours. A reversible ischemic neurologic deficit (RIND) is a fully reversible neurologic deficit that lasts more than 24 hours and less than 3 weeks. A stroke or permanent neurologic event lasts more than 3 weeks or causes death. Psychomotor deficits determined by specialized testing are not considered neurologic events related to operated valves. Patients who do not awaken or who awaken after operation with a new stroke are excluded in tabulations of valve-related morbidity.

A peripheral embolic event is an operative, autopsy, or clinically documented embolus that produces symptoms from complete or partial obstruction of a peripheral (noncerebral) artery. Patients who awake with a myocardial infarction are excluded. Patients who have a myocardial infarction after the perioperative period are also excluded unless a coronary arterial embolus is shown to be the cause of the infarction by operation, autopsy, or clinical investigation. Emboli proven to consist of nonthrombotic material (e.g., atherosclerosis, myxoma) are excluded.

Bleeding event (formerly anticoagulant-related hemorrhage). Any episode of major internal or external bleeding that causes death, hospitalization, or permanent injury (e.g., vision loss) or necessitates transfusion.
The complication bleeding event applies to all patients whether or not they are taking anticoagulants or antiplatelet drugs, since bleeding events can occur in patients who are not receiving anticoagulants. Embolic stroke complicated by bleeding is classified as a neurologic event under embolism and is not included as a separate bleeding event.

The warfarin anticoagulant status closest to the time that the patient has valve thrombosis, embolism, or a bleeding event should be reported in international normalized ratio units. Whether or not patients were receiving a platelet inhibitory drug (e.g., aspirin, dipyridamole) should also be reported.

Operated valvular endocarditis. Any infection involving an operated valve.

The diagnosis of operated valvular endocarditis is based on customary clinical criteria including an appropriate combination of positive blood cultures, clinical signs, and histologic confirmation of endocarditis at reoperation or autopsy. Morbidity associated with active infection, such as valve thrombosis, thrombotic embolus, bleeding event, or paravalvular leak, is included under this category and is not included in other categories of morbidity.

\section{Consequences of morbid events}

Reoperation. Any operation that repairs, alters, or replaces a previously operated valve.

The reasons for reoperation should be reported and may include reasons other than valve-related morbidity, such as recall, excessive noise, or incidental or prophylactic removal. Enzymatic or catheteraided therapy of valve-related morbidity is not considered reoperation, but the morbid event that prompted the intervention should be reported.

Valve-related mortality. Death caused by structural valvular deterioration, nonstructural dysfunction, valve thrombosis, embolism, bleeding event, operated valvular endocarditis, or death related to reoperation of an operated valve. Sudden, unexplained, unexpected deaths of patients with an operated valve are included as valve-related mortality. Deaths caused by heart failure in patients with advanced myocardial disease and satisfactorily functioning cardiac valves are not included. Specific causes of valve-related deaths should be designated and reported.

Sudden, unexpected, unexplained death. The cause of these deaths is unknown and the relationship to an operated valve is also unknown. Therefore, these deaths should be reported as a separate category of valve-related mortality if the cause cannot be determined by clinical data or autopsy. 
Cardiac death. All deaths resulting from cardiac causes. This category includes valve-related deaths (including sudden unexplained deaths) and nonvalve-related cardiac deaths (e.g., congestive heart failure, acute myocardial infarction, documented fatal arrhythmias).

Total deaths. All deaths resulting from any cause after a valve operation.

Permanent valve-related impairment. Any permanent neurologic or other functional deficit caused by structural valvular deterioration, nonstructural dysfunction, valve thrombosis, thrombotic embolism, bleeding event, operated valvular endocarditis, or reoperation.

\section{Data collection}

Data collection and reporting for all operated valves should include valve location (aortic, mitral, tricuspid, pulmonary, aortic and mitral, mitral and tricuspid) and category of operated valve (i.e., aortic pericardial bioprosthesis, fresh aortic allograft, mechanical mitral prosthesis, tricuspid ring annuloplasty). For prosthetic valves, including bioprostheses, the manufacturer and model should be reported with designation of different models and manufacturers. For allograft and xenograft valves the method of preservation should be given. Valve sizes for each category of valve, valve model, and valve location should be stated.

Additional pertinent material. In addition each report should specify:

1. The patient population from which the sample population was collected. The inclusive dates of operation and whether or not the series was consecutive should be stated. Criteria used to select patients should be specified and defined. If a subset of the sample population is reported, the total number of patients who received an operated valve during the same time frame should be reported.

2. The method employed for follow-up (e.g., examination, telephone, letter, retrospective review versus prospective study). The percentages of responses from each method should be given.

3. The time period (closing interval) required to complete current follow-up. (The closing interval, in which the present status of all patients is determined, should be as short as possible.)

4. The completeness of follow-up during the closing interval. (This should be $95 \%$ or better.)

5 . The percentage of autopsies and documented causes of death.
6. New York Heart Association functional class at the time of follow-up.

\section{Data analysis and reporting}

The method of reporting data should facilitate comparison between reports and support the conclusions, inferences, and predictions made. Some methods that may be useful are discussed below. The methods chosen to analyze the collected data will be influenced by the purpose of the report and the availability of various analytical techniques. Methods used in the collection and analysis of data should be defined in the Methods section by references or defined in an appendix.

As with cardiac surgery, statistical science is a dynamic discipline and the methods used may vary between statisticians. However, in all reports the conclusions, predictions, and inferences made should be supported by the collected data and appropriate analysis of the data.

Percentages (not time-related). Some morbid events that occur within a short time frame (such as the interval between operation and 30 days or hospital discharge) may be reported as a simple percentage, that is, the number of events divided by the number of patients (e.g., percent operative mortality). Percentages should be presented with confidence intervals ${ }^{6}$ and may be compared by $\chi^{2}$ analysis of Fisher's exact test. ${ }^{7}$ Logistic regression ${ }^{8}$ is available for evaluating the simultaneous influence of several risk factors on a dichotomous outcome variable (percentage) and is often used to establish a risk model, that is, a mathematical formula that incorporates such factors.

Time-related events. Most valve-related events should be reported in a time-related manner, with operation designated as time zero. Kaplan-Meier ${ }^{9}$ or other life table techniques ${ }^{10}$ provide actuarial estimates of morbid events and should be reported with the standard error of the estimate or with appropriate (usually $67 \%$ or $95 \%$ ) confidence limits. The number of patients remaining at risk should be indicated at appropriate intervals, and curves should not be extrapolated beyond time frames containing very few patients. Although comparisons between subsets of patients can be made, actuarial methods are not predictive beyond the time of the last actuarial estimate and cannot be adapted to multivariate analysis. These methods are called nonparametric or distribution-free because they do not assume a particular statistical distribution or model. 
The Cox proportional hazard model ${ }^{11}$ produces time-dependent analysis of valve-related events and provides a multivariable, stepwise regression method to identify risk factors associated with specific valve-related morbid events during specific time intervals. The Cox method is a semiparametric (model partly specified) approach, which makes no assumption about the shape of the underlying hazard function, but identifies risk factors and estimates multipliers of the baseline hazard that are the relative risks associated with the risk factors. Several methods are available for assessing the assumption of proportional hazards. ${ }^{12}$ The results of a multivariable analysis should be accompanied by a list of the variables considered and a tabular presentation of the numerical results.

A fully parametric method (model completely specified) of calculating a hazard function of valverelated morbid events defines the instantaneous risk of an event at any time after operation. ${ }^{13-16}$ Such methods permit univariate and multivariate analysis, provide predictive information beyond the time of the last event, indicate whether the risk is constant, and provide confidence limits.

Linearized rates. Some of the aforementioned methods have been extended to consider repeated events in the same patient, although the software is not widely available. A simple and widely used approach uses "linearized" rates (events per 100 patient-years or percent per year, calculated as the number of events divided by the total patient-years) to summarize the incidence of multiple events in individual patients. These rates should be considered only approximate unless the hazard function for the complication under study is constant during the entire time interval considered (which is often not true with regard to the early postoperative period) and unless the risk of recurrent events is the same as for initial events (which is often not the case). Linearized rates should be reported with confidence limits, which can be based on the Poisson distribution ${ }^{17}$ or on likelihood ratio methods for comparing the means of exponential distributions. ${ }^{11,18}$ Linearized rates can be compared using the likelihood ratio test ${ }^{15,16,19}$ or a test based on the F-statistic. ${ }^{11,16}$

Cumulative incidence. The hazard function for a given morbid event represents a potential risk; its realization as an actual occurrence is influenced by the competing risks of other events, such as death or explant, which may terminate the valve's use before the event being analyzed can occur. The usual actuarial estimates ${ }^{9,10}$ assume that such terminating events are eliminated; it may be useful to determine the actual probability of occurrence, often called the cumulative incidence, which is less than that estimated by the usual actuarial method. ${ }^{4}$

The Committee thanks all respondents who contributed to this document and especially Eugene H. Blackstone, $\mathrm{MD}$, who provided particularly valuable criticism and information.

\section{REFERENCES}

1. Edmunds LH Jr, Clark RE, Cohn LH, Miller DC, Weisel $\mathrm{RD}$. Guidelines for reporting morbidity and mortality after cardiac valvular operations. J Thorac Cardiovasc Surg 1988; 96:351-3.

2. Edmunds LH Jr, Clark RE, Cohn LH, Miller DC, Weisel RD. Guidelines for reporting morbidity and mortality after cardiac valvular operations. Ann Thorac Surg 1988;46:257-9.

3. Clark RE, Edmunds LH Jr, Cohn LH, Miller DC, Weisel RD. Guidelines for reporting morbidity and mortality after cardiac valvular operations. Eur J Cardiothorac Surg 1988;2; 293-5.

4. Grunkemeier GL, Jamieson WRE, Miller DC, Starr A. Actuarial versus actual risk of porcine structural valve deterioration. J Thorac Cardiovasc Surg 1994;108:709-18.

5. Bodnar E, Butchart EG, Bamford J, Besselaar AMPH, Grunkemeier GL, Frater RWM. Proposal for reporting thrombosis, embolism and bleeding after heart valve replacement. J Heart Valve Dis 1994;3:120-3.

6. Vollset SE. Confidence intervals for a binomial propoertion. Stat Med 1993;12:809-24.

7. Fleiss JL. Statistical methods for rates and proportions. 2nd ed. New York: John Wiley, 1981:321 pages.

8. Hosmer DW, Lemeshow S. Applied logistic regression. New York: John Wiley, 1989:307 pages.

9. Kaplan EL, Meier P. Nonparametric estimation from incomplete observatyions. J Am Stat Assoc 1958;53:457-481.

10. Cutler SJ, Ederer F. Maximum utilization of the life table method in analyzing survival. J Chron Dis 1958;8:699-712.

11. Cox DR, Oakes D. Analysis of survival data. London: Chapman \& Hall, 1984:193 pages.

12. Hess KR. Graphical methods for assessing violations of the proportional hazards assumption in Cox regression. Stat Med 1995;14:1707-23

13. Blackstone EH, Naftel DC, Turner ME Jr. The decomposition of time-varying hazard into phases, each incorporating a separate stream of concomitant information. J Am Stat Assoc 1986;81:615-24.

14. Cohen AC, Whitten BJ. Parameter estimation in reliability and survival models. New York: Marcel Dekker, 1988:394 pages.

15. Lawless JR. Statistical models and methods for lifetime data. New York: John Wiley, 1982;580 pages.

16. Gross AJ, Clark VA. Survival distributions: reliability applications in the biomedical sciences. New York: John Wiley, 1976:331 pages.

17. Sahai $\mathbf{H}$, Kurshid A. Confidence intervals for the mean of a Poisson distribution: a review. Biom J 1993;35:857-867.

18. Kalbfleisch JD, Prentice RL. The statistical analysis of fairure time data. New York: John Wiley, 1980:321 pages.

19. Lee ET. Statistical methods for survival data analysis. Belmont (CA): Lifetime Learning Publications, 1980:567 pages. 